\title{
Determining Low-Frequency Earth Return Impedance: A Consistent Electromagnetic Approach
}

\section{Karolina Kaszás-Lažetić, Dejana Herceg, Nikola Djurić, Miroslav Prša}

Faculty of Technical Sciences, University of Novi Sad

Trg D. Obradovića 6, 21000 Novi Sad, Serbia

e-mail: kkasas@uns.ac.rs, vuletic@uns.ac.rs,ndjuric@uns.ac.rs,prsa@uns.ac.rs

\begin{abstract}
In this paper, we present a new method for determining the earth return impedance. The proposed method, based on electromagnetic field theory, is very accurate and convenient for the analysis of earth behavior over a wide range of low frequencies. It takes into account all crucial physical events, including an exact treatment of the skin and proximity effects within the earth. Combining analytical and numerical procedures for finding the exact current distribution and the earth return impedance per unit length, we have developed an efficient and powerful tool that is described in this paper. In all calculations, real situations were considered, consisting of various soil resistivity values, overhead conductor heights, and combinations of parameters at various frequencies. Although most commonly applied simplified formulas give accurate results at industrial frequencies, significantly worse results are obtained in the presence of higher harmonics. Hence, verification of the developed method was achieved by comparing obtained results with the results of the Carson-Clem simplified formula and the FEM-based calculations. The generality of the developed program indicates that it may also be applied for calculating the earth return impedance in the presence of higher current harmonics.
\end{abstract}

Keywords: Applied electromagnetic approach; Bessel functions; current distribution; earth return impedance; Poynting vector flux

\section{Introduction}

During the transmission and distribution of electrical energy from production to consumption centers, there is a possibility of occurrence of current inside the earth. This current distribution may be caused by the applied low-frequency transmission system, as in the case of Single Wire Earth Return (SWER), or by ground faults, lightning strike, or utility overvoltage. Low frequency current distribution inside the earth appears also in the case of Through-The-Earth (TTE) 
communications. In all these cases, it is of great importance to find the current distribution within the earth and the earth's impedance.

Depending on the source of current distribution within the earth, three situations may be observed:

If the current distribution within the earth is produced by a low-frequency SWER system or if a low-frequency ground fault current is not momentarily eliminated, only low frequencies are present inside the earth. This implies that only current distribution at the basic industrial frequency and several higher harmonics should be investigated as a quasi-stationary case.

However, in a lightning strike or a situation when current inside the earth appears as a result of a sudden ground fault, the current very quickly varies in time and both electromagnetic and fast transients should be studied. In this case, electromagnetic wave theory should be applied to determine either Transverse Electromagnetic (TEM) or quasi TEM waves at frequencies up to several tenths of $\mathrm{MHz}$ [3], [13], [14], [15].

In the third case, in TTE communications, the earth impedance calculation is in the range of low frequencies. However, the problem is defined and treated by using the electromagnetic wave approach [4], [5].

These three cases are always investigated separately, although quasi-stationary and TEM or quasi TEM cases could be noticed during any ground fault.

In order to protect humans from electrocution as well as to optimize the elements of power transmission and distribution grounding systems, in this paper we only investigate the first case, low-frequency, quasi-stationary electromagnetic field.

A number of studies have been carried out aiming at evaluating the approximations derived from circuit theory, which are not appropriate. By using elements with lumped parameters (grounding resistors, capacitors, and inductances) to describe current behavior in the earth, many physical events cannot be taken into account. Thus, although such calculations are fairly accurate for basic frequencies, they are less accurate when analyzing higher harmonics.

To simplify the required mathematical apparatus, most approaches have used the concept of the simplified soil model. In this model, for example, the presence of the skin effect, especially for different values of earth resistivity, is concealed in the empiric formulas and diagrams usually used in power engineering.

The ground return parameters of low-frequency transmission lines have long been identified. In the technical literature, researchers have addressed the problem of calculating these parameters in a number of ways, which may be classified into three main groups. A detailed review of the presented methods is given in [25]. 
The first group includes papers dealing with Carson's fundamental method of calculating the earth return impedance [6] and improving it via additional correction terms [2], [10], [11], [17], [21], [26].

Papers where the image theory is applied to the complex depth of earth return conductors represent the next significant body of work [7], [8], [15].

The third group of papers deals with numerical approach manly based on the Finite Element Method (FEM) to calculate the ground return impedance [16], [22], [23], [24].

We describe here a new approach where the physical electromagnetic model is an essential starting-point. The applied mathematical methods are thus simply the consequence of the approach, enabling the problem to be solved successfully. Most of existing parameters, including ground resistivity, conductor height, and operational frequency, are taken into consideration. Initial results dealing with the current distribution are given in [19] and [20].

In order to verify the developed method, we also applied a numerical procedure based on the Finite Element Method (FEM). The results of both methods are presented and discussed in this paper and are compared with the results obtained via the Carson-Clem formula.

\section{Proposed Method}

In order to determine the earth's impedance, let us assume a system shown in Figure 1. The system is composed of an overhead conductor parallel to earth's surface, with the earth representing the return conductor.

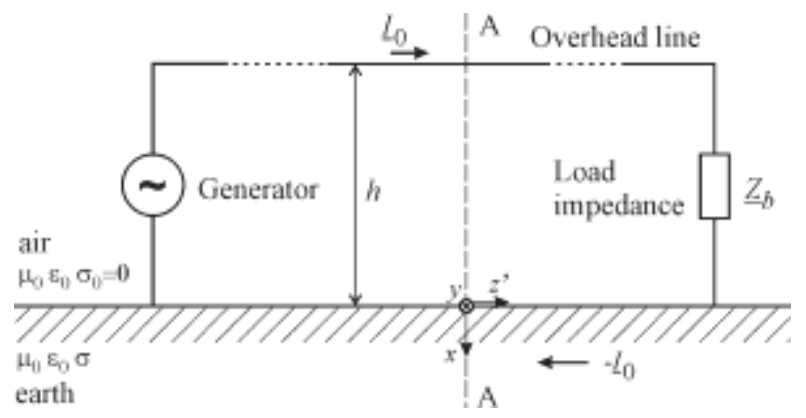

Figure 1

The principle of the earth as a return conductor

As a return conductor, the earth is assumed to have an infinite cross-section in conducting the current $-\underline{I}_{0}$ from the load impedance $\underline{Z}_{\mathrm{b}}$ towards the generator. 
However, due to the skin effect and the influence of the overhead conductor, the current density vector is not uniformly distributed across the infinite cross-section. Its maximal value appears under the overhead conductor and decreases with increasing distance. This maximal value depends on the following parameters: the height of the overhead conductor $h$, the current frequency $f$, permeability $\mu$, and the earth's conductivity $\sigma$.

In order to define the earth return impedance, the complex Poynting vector, $\underline{\boldsymbol{P}}=\underline{\boldsymbol{E}} \times \underline{\boldsymbol{H}}^{*}$, where $\underline{\boldsymbol{E}}$ is the complex electric field strength vector and $\underline{\boldsymbol{H}}^{*}$ is the conjugate complex magnetic field strength vector, should be determined. The Poynting's vector flux over the earth's surface, $S_{\mathrm{es}}$, presents the complex power transferred into the earth [18]:

$$
\underline{S}=P+j Q=\underline{Z}_{e}\left|\underline{I}_{0}\right|^{2}=\int_{S_{e s}}\left(\underline{\boldsymbol{E}} \times \underline{\boldsymbol{H}}^{*}\right) \cdot d \boldsymbol{S} .
$$

The earth return impedance per unit length, in all cases and at all frequencies, can be then be calculated as:

$$
\underline{Z}_{e}=\frac{1}{\left|\underline{I}_{0}\right|^{2}} \int_{S_{e s}}\left(\underline{\boldsymbol{E}} \times \underline{\boldsymbol{H}}^{*}\right) \cdot d \boldsymbol{S} .
$$

Calculation of magnetic field strength vector requires determining the current distribution within the homogenous earth.

\subsection{Determination of Electric Field Strength Vector within the Earth}

A geometric representation of the problem defined by cross section A - A shown in Figure 1 is presented in Figure 2.

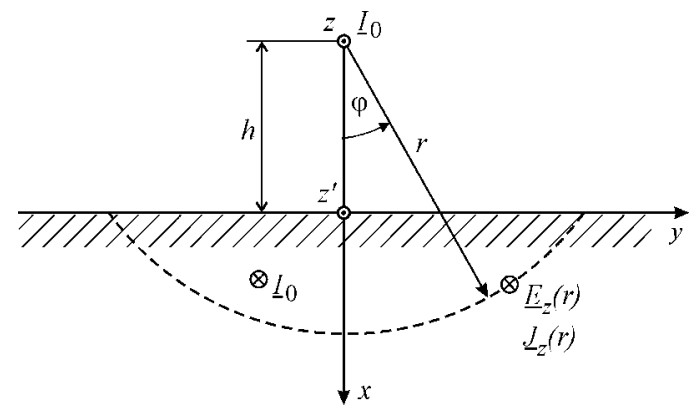

Figure 2

Geometry of the treated problem 
The geometry suggests that the cylindrical coordinate system $(r, \varphi, z)$ should be applied, where the $z$-axis passes through the axis of the overhead conductor. In this case, the current density vector has only a $z$ component that depends only on radius $r$.

A plane surface of the homogeneous earth, involved in applied cylindrical coordinate system, may provoke certain geometry problems. For this reason, the introduction of an additional Cartesian coordinate system $(x, y, z)$, shown in Figure 2, is a convenient solution to the Poynting vector flux calculation.

The entire calculation may be performed starting from the first two Maxwell equations in a complex domain for a quasi-stationary electromagnetic field:

$$
\begin{aligned}
& \nabla \times \underline{\boldsymbol{E}}=-j \omega \underline{\boldsymbol{B}} \\
& \nabla \times \underline{\boldsymbol{H}}=\underline{\boldsymbol{J}}
\end{aligned}
$$

From (1) and (2), the electric field strength vector in the earth is a solution to the partial differential equation in the cylindrical coordinate system [18]:

$\frac{\partial^{2} \underline{E}_{z}}{\partial r^{2}}+\frac{1}{r} \frac{\partial \underline{E}_{z}}{\partial r}-\underline{k}^{2} \underline{E}_{z}=0$

where the complex constant $\underline{k}^{2}$ is defined as:

$\underline{k}^{2}=\frac{j \omega \mu \sigma}{\pi} \arccos \frac{h}{r}$

In a quasi-stationary case, earth permittivity does not influence the electric field distribution within the earth, implying that only the earth permeability $\mu$ and earth conductivity $\sigma$ appear in (4). When the coefficient $\underline{k}^{2}$ is constant, the above equation is Bessel's equation with the solution:

$$
\underline{E}_{z}(r)=\underline{A}_{0}(\underline{k r})+\underline{B} K_{0}(\underline{k r}),
$$

where function $I_{0}(\underline{k r})$ is the modified Bessel function of the first kind of zero order, $K_{O}(\underline{\mathrm{k} r})$ is the modified Bessel function of the second kind of zero order, and $\underline{A}$ and $\underline{B}$ are arbitrary complex constants.

The proper function to represent $\underline{E}_{z}(r)$ is one that vanishes at $r \rightarrow \infty$, since it is well-known that the electric field intensity within the earth should approach zero with increasing radius. Therefore, the Bessel function of the first kind is discarded because of its infinite value when radius $r$ is infinitely large. As a consequence, the complex constant $\underline{A}$ is zero and the final solution for complex electric field strength vector is:

$$
\underline{E}_{z}(r)=\underline{B} K_{0}(\underline{k} r) \text {. }
$$


Function $K_{0}(\underline{k} r)$ is divided into real and imaginary parts:

$\underline{E}_{z}(r)=\underline{B}[\operatorname{ker}(a r)+j k e i(a r)]$

where $a$ is:

$a=\sqrt{\frac{\omega \mu \sigma}{\pi} \arccos \frac{h}{r}}$.

The complex constant $\underline{B}$ depends on frequency $f$, conductor's height above the earth $h$, and the earth's resistivity value $\rho$. It is determined by integrating the current density vector over the entire earth's cross-section $S_{\text {ecs }}(x-y$ plane), which must give the complex imposed current value:

$\underline{I}_{0}=\int_{S_{e c s}} \underline{\boldsymbol{J}} \cdot d \boldsymbol{S}$

\subsection{Current Density Vector Calculation}

The complex current density vector $\underline{\boldsymbol{J}}$ has the same direction as the complex electric field strength vector $\underline{\boldsymbol{E}}$. Hence, the former can be determined easily as:

$\underline{\boldsymbol{J}}=\sigma \underline{\boldsymbol{E}} \quad \underline{\boldsymbol{J}}_{z}=\sigma \underline{E}_{z}$.

In the entire calculation, the earth is treated as a homogeneous medium with constant conductivity to an infinite depth. This assumption is acceptable in cases where the earth acts as a return conductor [12].

\subsection{Determination of the Complex Magnetic Field Strength Vector}

Much more difficult is the determination of the complex magnetic field strength vector $\underline{\boldsymbol{H}}$, which is composed of two contributions: magnetic field due to the overhead conductor current and magnetic field produced by the current inside the earth.

\subsubsection{Determination of the Magnetic Field Produced by the Current in the Overhead Conductor}

The first contribution is given by the current $\underline{I}_{0}$ in the overhead conductor. Denoted by $\underline{\boldsymbol{H}}_{0}$, according to Figure 3 , this portion of the magnetic field strength vector at an arbitrary chosen point on the earth's surface, $\mathrm{T}(0, y)$, is:

$\underline{\boldsymbol{H}}_{0}=\frac{\underline{I}_{0}}{2 \pi r} \cdot \boldsymbol{i}_{\varphi}$. 


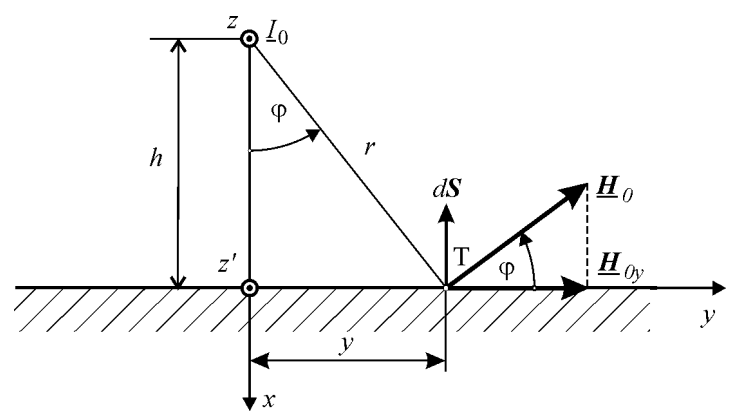

Figure 3

Magnetic field due to the overhead conductor

According to (1), in our case only its $y$ component is relevant:

$\underline{\boldsymbol{H}}_{0 y}=\frac{\underline{I}_{0}}{2 \pi r} \cos \varphi \cdot \boldsymbol{i}_{\varphi}=\frac{\underline{I}_{0}}{2 \pi} \frac{h}{h^{2}+y^{2}} \cdot \boldsymbol{i}_{\varphi}$

if we consider that

$\cos \varphi=\frac{h}{r} \quad$ and $\quad r^{2}=h^{2}+y^{2}$.

\subsubsection{Determination of the Magnetic Field Produced by the Current within the Earth}

The second contribution to the magnetic field strength vector is produced by the current density $\underline{J}_{z}(r)$ within the earth. If we denote this part of the magnetic field strength vector as $\underline{\boldsymbol{H}}_{e y}$, the resultant vector $\underline{\boldsymbol{H}}_{y}$ is the sum:

$\underline{\boldsymbol{H}}_{y}=\underline{\boldsymbol{H}}_{0 y}+\underline{\boldsymbol{H}}_{e y}$.

The second part of the magnetic field strength vector is much more difficult to evaluate.

For this calculation, let us consider the complex polar coordinates, shown in Figure 4 where $i_{r}$ represents the radial unit complex vector and $\boldsymbol{i}_{\varphi}$ represents the angular unit complex vector.

For easier procedure, in all further calculation the unit complex vectors are represented by their exponential forms: $\boldsymbol{i}_{r}$ is substituted by $e^{i \varphi}$ and $\boldsymbol{i}_{\varphi}$ is denoted as $i e^{i \varphi}$. 


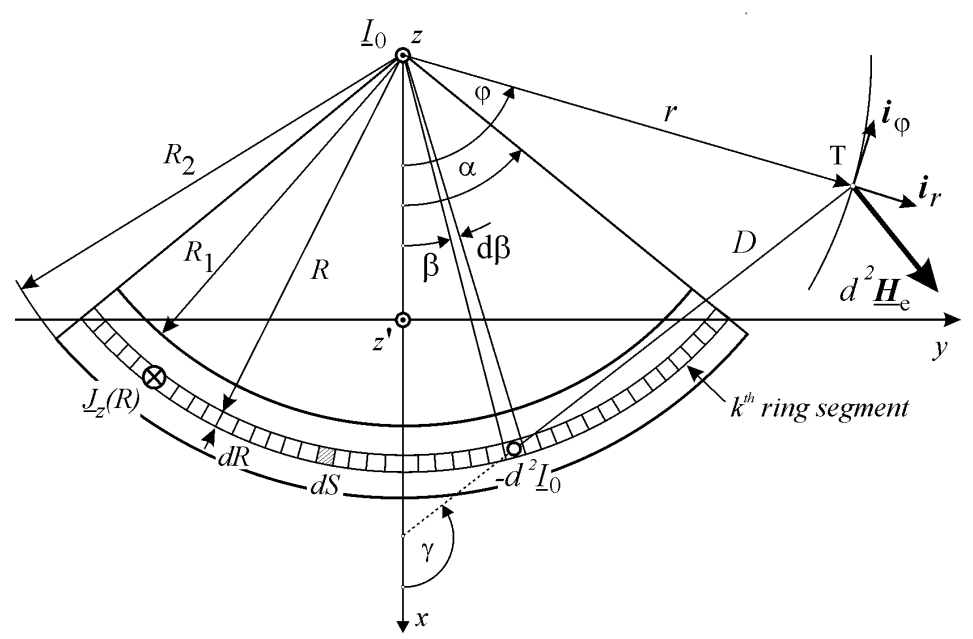

Figure 4

Magnetic field due to current within the earth

We use the imaginary unit $i=\sqrt{-1}$ because the unit $j=\sqrt{-1}$ was already used for the time dependence in the frequency domain.

The current filament $-d^{2} \underline{I}_{0}$ at distance $D$ at an arbitrary point $\mathrm{T}$ above the earth generates the magnetic field strength vector:

$d^{2} \underline{\boldsymbol{H}}_{e}=-i e^{i \gamma} \frac{d^{2} \underline{I}_{0}}{2 \pi D}=-i \frac{d^{2} \underline{I}_{0}}{2 \pi} \cdot \frac{1}{D e^{-i \gamma}}$

where

$d^{2} \underline{I}_{0}=\underline{J}_{z}(R) d S=\underline{J}_{z}(R) d \beta d R$

and

$D e^{i \gamma}=r e^{i \varphi}-R e^{i \beta} \quad$ or $\quad D e^{-i \gamma}=r e^{-i \varphi}-R e^{-i \beta}$.

In order to calculate Poynting vector flux through the ground surface, the point $\mathrm{T}$ has to drop onto the earth's surface, as shown in Figure 3.

Hence, the magnetic field strength vector is:

$$
d^{2} \underline{\boldsymbol{H}}_{e}=-i \frac{\underline{J}_{z}(R)}{2 \pi} R \frac{d \beta}{r e^{-i \varphi}-\mathrm{Re}^{-i \beta}} d R=-i \frac{J_{z}(R)}{2 \pi} \frac{R}{r} \frac{e^{i \varphi} d \beta}{1-\frac{\mathrm{R}}{r} e^{i \varphi} \mathrm{e}^{-i \beta}} d R .
$$


Expanding the expression $\left(1-\frac{\mathrm{R}}{r} e^{i \varphi} \mathrm{e}^{-i \beta}\right)^{-1}$ into an infinite series:

$$
\left(1-\frac{\mathrm{R}}{r} e^{i \varphi} \mathrm{e}^{-i \beta}\right)^{-1}=\sum_{m=0}^{\infty}\left(\frac{R}{r}\right)^{m} e^{i m \varphi} e^{-i m \beta}
$$

the magnetic field strength vector at point $\mathrm{T}$ produced by the current through a ring segment of cross-section $\alpha\left(R_{2}{ }^{2}-R_{1}{ }^{2}\right)$, is:

$$
\delta \underline{\boldsymbol{H}}_{e}=-i \frac{1}{\pi} \sum_{m=0}^{\infty} \frac{\sin m \alpha}{m} e^{\mathrm{i}(m+1) \varphi}\left[\int_{R_{1}}^{R_{2}}\left(\frac{R}{r}\right)^{m+1} \underline{J}_{z}(R) d R\right] .
$$

This vector has two components:

$$
\delta \underline{\boldsymbol{H}}_{e}=\boldsymbol{i}_{r} \delta \underline{H}_{e r}+\boldsymbol{i}_{\varphi} \delta \underline{H}_{e \varphi}=e^{i \varphi} \delta \underline{H}_{e r}+i e^{i \varphi} \delta \underline{H}_{e \varphi} .
$$

The magnetic field strength vector components are:

$$
\begin{aligned}
& \delta \underline{H}_{e r}=\frac{1}{\pi} \sum_{m=0}^{\infty}\left[\frac{\sin m \alpha}{m} \sin m \varphi \int_{R_{1}}^{R_{2}}\left(\frac{R}{r}\right)^{m+1} \underline{J}_{z}(R) d R\right], \\
& \delta \underline{H}_{e \varphi}=-\frac{1}{\pi} \sum_{m=0}^{\infty}\left[\frac{\sin m \alpha}{m} \cos m \varphi \int_{R_{1}}^{R_{2}}\left(\frac{R}{r}\right)^{m+1} \underline{J}_{z}(R) d R\right] .
\end{aligned}
$$

Finally, according to Figure 4, due to the small thickness of the ring segment, the linear change of current density vector inside the segment is:

$$
J_{z}(R)=a R+b, \quad \text { for } \quad R_{1} \leq R \leq R_{2} .
$$

From the numerical values $J_{z}\left(R_{1}\right)$ and $J_{z}\left(R_{2}\right)$, the constants $a$ and $b$ are calculated as:

$$
a=\frac{J_{z}\left(R_{2}\right)-J_{z}\left(R_{1}\right)}{R_{2}-R_{1}}, \quad b=J_{z}\left(R_{1}\right)-\mathrm{aR}_{1}=J_{z}\left(R_{2}\right)-\mathrm{aR}_{2} .
$$

Hence, the components of the magnetic field strength vector(16) are:

$$
\begin{aligned}
\delta \underline{H}_{e r} & =\frac{1}{\pi} \sum_{m=0}^{\infty} \frac{\sin m \alpha}{m} \sin m \varphi \frac{a r^{2}}{m+3}\left[\left(\frac{R_{2}}{r}\right)^{m+3}-\left(\frac{R_{1}}{r}\right)^{m+3}\right]+ \\
& +\frac{1}{\pi} \sum_{m=0}^{\infty} \frac{\sin m \alpha}{m} \sin m \varphi \frac{b r}{m+2}\left[\left(\frac{R_{2}}{r}\right)^{m+2}-\left(\frac{R_{1}}{r}\right)^{m+2}\right]
\end{aligned}
$$




$$
\begin{aligned}
\delta \underline{H}_{e \varphi}= & -\frac{1}{\pi} \sum_{m=0}^{\infty} \frac{\sin m \alpha}{m} \cos m \varphi \frac{a r^{2}}{m+3}\left[\left(\frac{R_{2}}{r}\right)^{m+3}-\left(\frac{R_{1}}{r}\right)^{m+3}\right]- \\
& -\frac{1}{\pi} \sum_{m=0}^{\infty} \frac{\sin m \alpha}{m} \cos m \varphi \frac{b r}{m+2}\left[\left(\frac{R_{2}}{r}\right)^{m+2}-\left(\frac{R_{1}}{r}\right)^{m+2}\right]
\end{aligned}
$$

The expressions under the sum signs were calculated for each $k^{\text {th }}$ ring segment shown in Figure 4. Instead of infinite sums, the sums were performed on $n$ chosen ring segments. The $y$ component of the magnetic field strength vector due to the current within $n$ ring segments inside the earth can be expressed as:

$$
\underline{H}_{e y}=\sum_{k=1}^{n}\left[\left(\delta \underline{H}_{e r k}\right)_{y}+\left(\delta \underline{H}_{e \varphi k}\right)_{y}\right] \text {. }
$$

\subsubsection{Determination of the Total Magnetic Field Strength Vector}

The total $y$ component of the magnetic field strength vector is:

$$
\underline{H}_{y}=\underline{H}_{0 y}+\underline{H}_{e y}=\frac{\underline{I}_{0}}{2 \pi} \frac{h}{h^{2}+y^{2}}+\sum_{k=1}^{n}\left(\delta \underline{H}_{e r k} \frac{y}{\sqrt{h^{2}+y^{2}}}+\delta \underline{H}_{e \varphi k} \frac{h}{\sqrt{h^{2}+y^{2}}}\right) \text {. }
$$

Knowing both corresponding components: the $z$ component of the complex electric field strength vector $\underline{E}_{z}$ and the $y$ component of the complex magnetic field strength vector $\underline{H}_{y}$, we can calculate the complex Poynting vector and its flux over the earth's surface:

$$
\underline{S}=\underline{Z}_{e}\left|\underline{I}_{0}\right|^{2}=\int_{S_{e s}}\left(\underline{E}_{z} \underline{H}_{y}^{*}\right) \cdot d S .
$$

According to (1), the earth return impedance can be calculated as:

$$
\underline{Z}_{e}=\frac{1}{\left|\underline{I}_{0}\right|^{2}} \int_{S_{e S}}\left(\underline{E}_{z} \underline{H}_{y}^{*}\right) \cdot d S \text {. }
$$

\section{Electromagnetic Field Calculation by Applying FEM}

FEM is a well-known and powerful tool designed to solve many research problems in electrical engineering theory and practice. It is a numerical method widely used to calculate different electromagnetic field problems. In this paper, 
FEM has been applied to justify the validity of the results obtained by the proposed method used to calculate the earth return impedance.

The FEM formulation proposed in this paper provides two distinct approaches for calculating the earth resistance and earth inductance (reactance). For both, the same model shown in Figure 1 and Figure 2 may be used. The entire numerical calculation may be carried out for the same cross-section A-A and the problem could be treated as two-dimensional case.

In order to determine the distribution of the magnitude of the current density vector within the earth, the most convenient way is to solve the scalar complex partial differential equation:

$$
\frac{\partial^{2} \underline{J}_{z}}{\partial x^{2}}+\frac{\partial^{2} \underline{J}_{z}}{\partial y^{2}}-\underline{k}^{2} \underline{J}_{z}=0
$$

where $J_{z}$ is the $z$ component of the complex imposed current density vector, and $\underline{k}^{2}$ is defined in (4).

The resistive power losses per unit length, which result in heat dissipation inside the earth, are defined as:

$$
P_{J}^{\prime}=\frac{P_{J}}{\ell}=\frac{1}{\ell} \int_{V_{\text {earth }}} \rho\left|\underline{J}_{z}\right|^{2} d v=\frac{1}{\ell} \int_{S_{\text {ecs }}} \rho\left|\underline{J}_{z}\right|^{2} \ell d S=\int_{S_{\text {ecs }}} \rho\left|\underline{J}_{z}\right|^{2} d S
$$

where $\ell$ is the length of the calculation domain along $z$ axis. The earth resistance per unit length may be easily obtained from:

$$
R^{\prime}=\frac{P_{J}^{\prime}}{\left|\underline{I}_{0}\right|^{2}}
$$

For calculating the earth inductance, it is necessary to determine magnetic energy per unit length stored in the magnetic field within the earth:

$$
W_{m}^{\prime}=\frac{W_{m}}{\ell}=\frac{1}{2 \ell} \int_{V_{\text {earth }}} \mu|\underline{H}|^{2} d v=\frac{1}{2} \int_{S_{\text {ecs }}} \mu|\underline{H}|^{2} d S .
$$

Then, the earth inductance per unit length may be easily derived from (22):

$$
L_{\text {earth }}^{\prime}=\frac{2 W_{m}^{\prime}}{\left|\underline{I}_{0}\right|^{2}} \text {. }
$$

The calculated result is only the first contribution to the system's inductance. The second contribution, representing the overhead conductor's inner inductance per unit length, should be added to (23):

$$
L^{\prime}=L_{\text {earth }}^{\prime}+L_{\text {in }}^{\prime}=L_{\text {earth }}^{\prime}+\frac{\mu_{0}}{8 \pi} .
$$




\section{Results and Discussion}

We calculate the current distribution by applying all three methods over a homogeneous earth for four different values of earth resistivity, five values of overhead conductor height, and nine frequencies. The results are presented in Table 1.

Table 1

Input parameters

\begin{tabular}{|c|c|c|c|c|c|c|c|c|c|}
\hline$\rho[\Omega \cdot \mathrm{m}]$ & 50 & 250 & 1,000 & 2,500 & & & & & \\
\hline$h[\mathrm{~m}]$ & 10 & 15 & 20 & 25 & 30 & & & & \\
\hline$f[\mathrm{~Hz}]$ & 50 & 100 & 150 & 250 & 350 & 450 & 750 & 1,500 & 2,500 \\
\hline
\end{tabular}

Since most soil types are non-magnetic, we assume that the relative permeability of the earth is equal to unity, with the relative permittivity also considered equal to one.

The Bessel function values (3) were found in [1]. Frequencies ranging from $50 \mathrm{~Hz}$ to $2,500 \mathrm{~Hz}$ were examined. The sinusoidal current assumed in the overhead conductor is presented in the complex domain as:

$\underline{I}=(1+j 0) A$.

In the 2D FEM model, the quasi-stationary approximation is valid for the explored frequency range. Calculation of the earth return impedance using FEM requires a definition of appropriate boundaries. The observed area was chosen to be a square with the side large enough to neglect the current on and outside the boundary.

The large number of elements far away from the overhead conductor has no impact on the accuracy of the calculation. However, they require substantial computational effort. Most changes in the current distribution appear just below the conductor and in its vicinity. In order to reduce the number of finite elements and to emphasize the region near the overhead conductor without decreasing the accuracy of the calculation, a manual mesh generation is applied. The suggested approach enabled a compromise between the two opposing requirements: the substantial decrease in the number of finite elements and improving accuracy of the calculation.

\subsection{Current Distribution}

The current distribution along the $x$ axis $(y=0)$ shown in Figure 4, within the homogeneous ground at $\rho=50 \Omega \cdot \mathrm{m}$ and $f=50 \mathrm{~Hz}$, for five different conductor heights, calculated using the proposed method based on complex Poynting vector flux, is shown in Figure 5. 


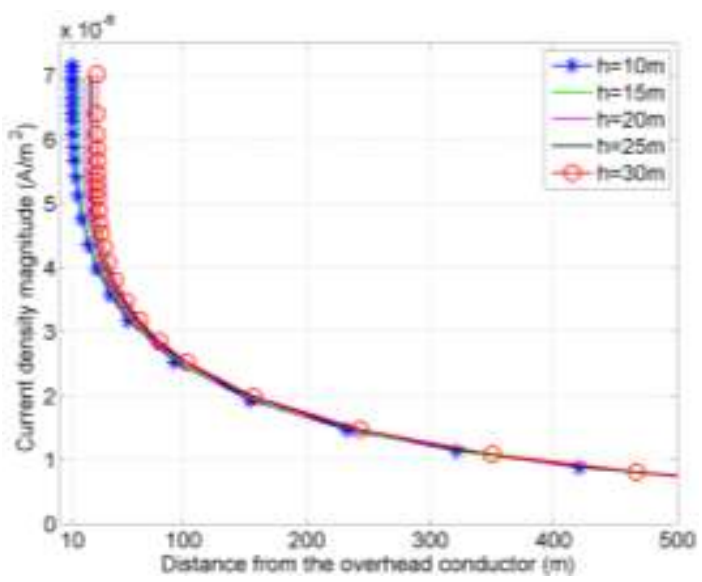

Figure 5

Magnitude of current density vector as a function of the overhead conductor height

The figure reveals that the influence of conductor height on current distribution within the earth is negligible for the same value of soil resistivity.

Magnitudes of current density vector for four different homogeneous earth resistivity values and for a single conductor height of $h=15 \mathrm{~m}$ at $f=50 \mathrm{~Hz}$ are presented in Figure 6.

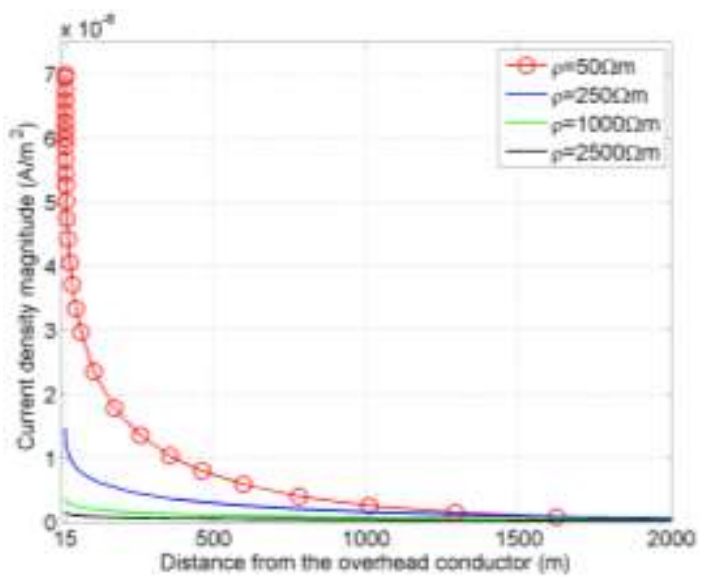

Figure 6

Magnitude of current density vector for $h=15 \mathrm{~m}, f=50 \mathrm{~Hz}$ and four different earth resistivity values

It is evident from Figure 6 that the skin effect is most noticeable in the case of low resistivity value of $\rho=50 \Omega \cdot \mathrm{m}$. Hence, for all four ground resistivity values, the current density is largest on the earth's surface and decreases rapidly with distance from the conductor above. 
In the three other cases for higher soil resistivity values, the skin effect is less present and the penetration depth is much higher.

\subsection{Calculation of Earth Return Impedance}

In order to verify the proposed method and calculated impedances, the results were compared with the results obtained by a numerical procedure based on FEM and, the Carson's (Carson-Clem) formula [9], [27] for ground current impedance:

$\underline{Z}^{\prime}=\left(R_{c}^{\prime}+9,87983 \cdot 10^{-4} f+j 2.8937 \cdot 10^{-3} f \cdot \log \frac{658.8 \sqrt{\frac{\rho}{f}}}{G M R}\right)[\Omega / \mathrm{km}]$

where:

$R_{c}^{\prime}$ is the resistance per unit length of the overhead conductor in ohms per kilometre $[\Omega / \mathrm{km}]$,

$\rho$ is the earth resistivity in ohm-meters $[\Omega \cdot \mathrm{m}]$,

$f$ is the frequency in hertz and

GMR is the effective radius of the overhead conductor in meters.

Earth resistance per unit length, calculated based on the developed code for the earth resistivity $\rho=50 \Omega \cdot \mathrm{m}$ and five different heights of the overhead conductor, is shown in Figure 7. The results obtained via (25), labeled "Carson", do not depend on the conductor's height. The results using the numerical procedure (FEM) are obtained for the conductor's height $h=15 \mathrm{~m}$.

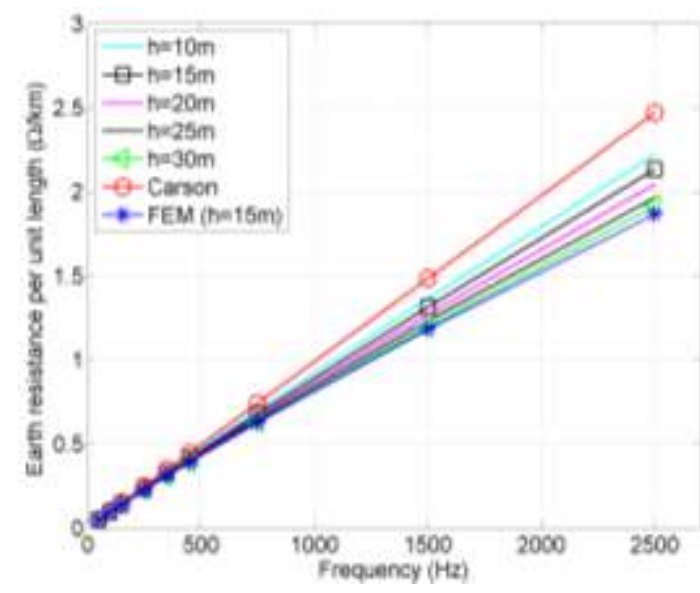

Figure 7

Earth resistance per unit length for $\rho=50 \Omega \cdot \mathrm{m}$ and five different heights of the overhead conductor 
Figure 7 illustrates a good agreement among all resistance calculations at frequencies up to tenth harmonic, which validates the proposed method. It also shows that simplified Carson-Clem formula (25) can be applied as well for finding accurate resistance per unit length at industrial frequencies and lower harmonics (up to tenth harmonic).

Again, the influence of conductor height is less significant at lower frequencies while it increases at higher frequencies.

The relationship between frequency and the earth's resistance per unit length for a conductor height of $h=15 \mathrm{~m}$ and four different earth resistivity values, calculated by applying the proposed method is shown in Figure 8 . The resistance per unit length using the Carson-Clem formula does not include the resistivity value. The FEM calculation was performed with the resistivity value $\rho=50 \Omega \cdot \mathrm{m}$.

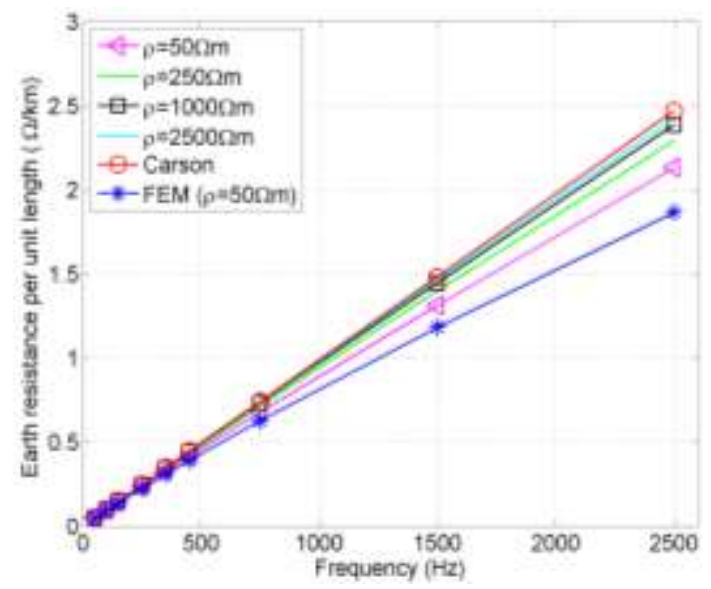

Figure 8

Earth resistance per unit length for $h=15 \mathrm{~m}$ and four different values of earth resistivity

The influence of earth resistivity is greater than conductor height, especially at higher frequencies, as shown in Figure 8. Good agreement of all results is evident.

The influence of frequency on earth's reactance per unit length, for a constant conductor height of $h=15 \mathrm{~m}$ and four different earth resistivity values is shown in Figure 9. The results labeled "Carson" are obtained via (25), at $\rho=50 \Omega \cdot \mathrm{m}$, with a conductor radius of $r_{\mathrm{s}}=0.001144 \mathrm{~m}$ are shown as well along with numerical results (FEM). At $\rho=50 \Omega \cdot \mathrm{m}$, very good agreement can be seen between the proposed method and the numerical FEM procedure. However no agreement is achieved using the Carson-Clem formula (25).

Figure 9 reveals that the earth's reactance per unit length does not increase linearly but rather slowly with increasing frequency. This indicates that earth's inductance per unit length decreases with increasing frequency. 


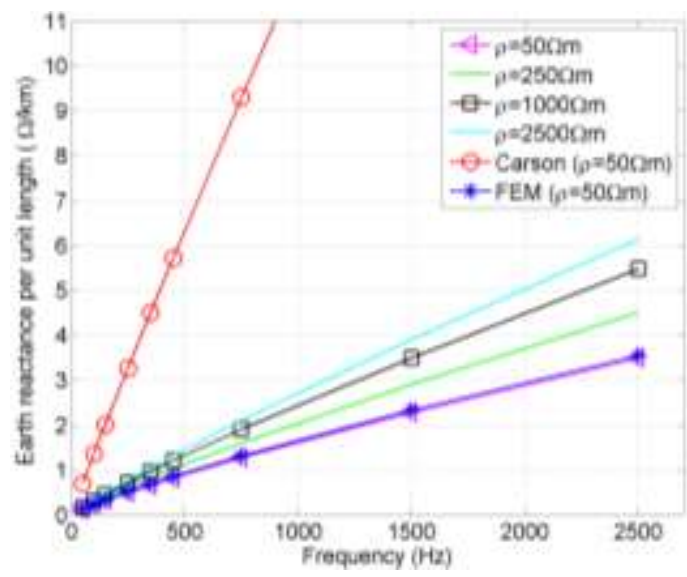

Figure 9

Earth reactance per unit length for $h=15 \mathrm{~m}$ and four different values of earth resistivity

The dependence of the earth's inductance per unit length on frequency for a constant conductor height of $h=15 \mathrm{~m}$ and for four values of earth resistivity is shown in Figure 10.

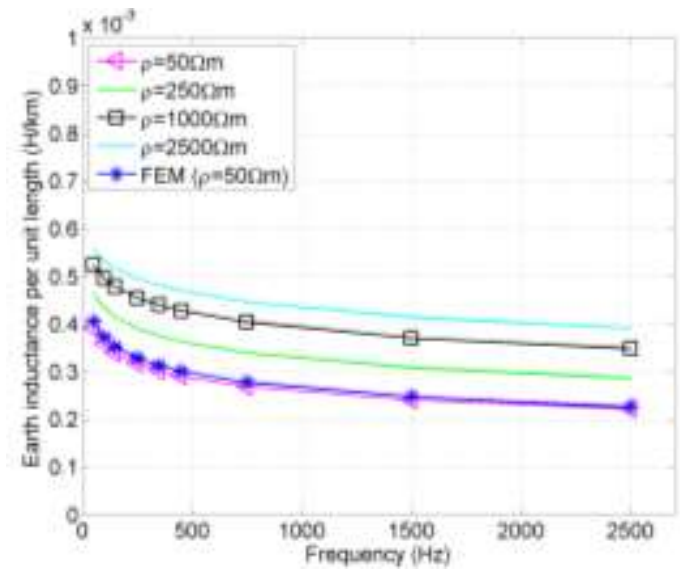

Figure 10

Earth inductance per unit length for $h=15 \mathrm{~m}$ and four values of earth resistivity

As expected, the earth's inductance per unit length decreases with increasing frequency and increasing skin effect.

At the highest earth resistivity, the skin effect is almost negligible and the earth's inductance per unit length reaches the highest values and then decreases slowly with increasing frequency. In contrast, at the lowest earth resistivity and with a more significant skin effect, the earth's inductance per unit length reaches minimum and decreases with increasing frequency. 
An excellent agreement between the results obtained by the proposed method and results obtained by FEM is shown in Figure 10.

The inductances per unit length calculated using the Carson-Clem formula (25) reaches much higher values, with the same tendency. These values are not shown in Figure 10.

\section{Conclusions}

In this paper, we present a novel procedure with which to determine earth return impedance, based on an electromagnetic approach. The suggested method is very convenient for the accurate calculation of earth's impedance and offers an exact treatment of the skin effect within the earth.

The results show that the suggested procedure provides a more accurate estimate of current distribution within the earth when higher harmonics are present then most applied simplified formulae. Moreover, the procedure also correctly calculates the electric and magnetic fields both in the ground and in the space between the conductor and the earth's surface, which is the space of transmitting energy.

Knowledge of the earth's impedance is essential when building a reliable equivalent circuit of any transmission system. Therefor, the developed method represents an efficient tool for calculation of impedance in electrical power transmission and distribution systems that include earth return where ground currents are of particular significance. Such applications include safety analysis and calculation of numerous physical variables that appear within and on the earth's surface. The method may also be a powerful tool for investigating the most common, single line-to-ground (SLG) fault cases.

\section{Acknowledgement}

This project was supported by the Ministry of Science and Technology Development, Republic of Serbia, under the grant project TR 32055.

\section{References}

[1] Abramowitz M. and Stegun I. A., "Bessel Functions of Integer Order," in Handbook of Mathematical Functions, $9^{\text {th }}$ Ed. New York, NY: Dover Publications, 1970, pp. 355-433

[2] Alvarado F. L. and Betancourt R., "An Accurate Closed-form Approximation for Ground Return Impedance Calculations," 1983, Proc. IEEE, Vol. 71, pp. 279-280

[3] Arnautovski-Toseva V. and Grcev L., "High Frequency Current Distribution in Horizontal Grounding Systems in Two-Layer Soil" in Proc. 2003 International Symposium on Electromagnetic Compatibility, pp. 205208 
[4] Bataller V., Muñoz A., Molina Gaudó P., Mediano A., Cuchí J. A. and Villarroel J. L. "Earth Impedance Model for Through-the-Earth Communication Applications with Electrodes" Radio Science, 2010, Vol. 45, RS6015, pp. 1-18

[5] Bataller V., Muñoz A., Molina Gaudó P., Mediano A., Cuchí J. A. and Villarroel J. L. "Electrode Contact Impedance Measurement in Throughthe-Earth Communication" in Proc. $200914^{\text {th }}$ International Conference on Design of Circuits and Integrated Systems, pp. 1-6

[6] Carson J. R., "Wave Propagation in Overhead Wires with Ground Return," Bell. Syst. Tech. J., 1926, Vol. 5, pp. 539-554

[7] Deri A. and Tevan G., "Mathematical Verification of Dubanton's Simplified Calculation of Overhead Transmission Line Parameters and Its Physical Interpretation," Archiv für Elektrotechnik, 1981, Vol. 63, pp. 191198

[8] Deri A., Tevan G., Semlyen A. and Castanheira A., "The Complex Ground Return Plane: a Simplified Model for Homogeneous and Multi-Layer Earth Return" IEEE Trans. Power App. Syst., 1981, Vol. PAS-100, No. 8, pp. 3686-3693

[9] Ebrahimi R., Babaee A. and Hoseynpoor M., "Evaluation and Calculation of Overhead Line Impedance in Distribution Networks", Australian J. Basic Appl. Sci., 2011, Vol. 5, No. 8, pp. 1278-1284

[10] Hofmann L., "Series Expansions for Line Series Impedances Considering Different Specific Resistances, Magnetic Permeabilities, and Dielectric Permittivities of Conductors, Air, and Ground," IEEE Trans. Power Del., 2003, Vol. 18, No. 2, pp. 564-570

[11] Leaños O. R., Naredo J. L. and Moreno P., "Assessment of Approximate Formulas for Calculating Overhead-Line Earth-Impedances," in 2008 Proc. Power Symposium, NAPS'08, pp. 1-6

[12] Micu D. D., Czumbil L., Prsa M. and Kasas-Lazetic K., "Interfstud Electromagnetic Interference Software - An Accurate Evaluation of Current Distribution in Soil and in Underground Pipelines," in Proc. 2012 International Symposium on Electromagnetic Compatibility, Rome 2012, pp. 1-5

[13] Olsen R. G and Pankaskie T. A., "On the Exact, Carson and Image Theories for Wires at or above the Earth's Interface," IEEE Trans. Power. App. Syst., Vol. PAS-102, 1983, No. 4, pp. 769-778

[14] Olsen R. G. and Willis M. C., "A Comparison of Exact and Quasi-Static Methods for Evaluating Grounding Systems at High Frequencies", IEEE Trans. Power Del., 1996, Vol. 11, No. 2, pp. 1071-1080 
[15] Papadopoulos T. A., Papagiannis G. K. and Labridis D. A., "Wave Propagation Characteristics of Overhead Conductors above Imperfect Stratified Earth for a Wide Frequency Range”, IEEE Trans. Power Del., 2009, Vol. 45, No. 3, pp. 1064-1067

[16] Papagiannis G. K., Tsiamitros A., Labridis D. P. and Dokopoulos P. S., “A Systematic Approach to the Evaluation of the Influence of Multilayered Earth on Overhead Power Transmission Lines," IEEE Trans. Power Del., 2005, Vol. 20, No. 4, pp. 2594-2601

[17] Pollaczek F., "Über das feld einer unendlich langen wechselstromdurchflossenen Einfachleitung", Elektrische Nachrichten Technik, 1926, Vol. 3, No. 9, pp. 339-359

[18] Popović Z. and Popović B. D., "Maxwell's Equations" in Introductory Elektromagnetics, Upper Saddle River, New Jersey 07458: Prentice Hall, 1999, pp. 359-382

[19] Prsa M. and Kasas-Lazetic K., "Magnetic Field due to a Current Distribution within the Earth," in Proc. 2008 Conf. on Electricity Distribution of Serbia, CIRED, pp. 71-75

[20] Prša M. and Kasaš-Lažetić K., "An Accurate Determination of Current Distribution within the Earth," in Proc. 2007 International Conf. on Applied Electromagnetics PES 2007, Paper No. 07-9, pp. 1-4

[21] Ramirez A. and Uribe F., "Assessment of Approximate Formulas for Calculating Overhead-Line Earth-Impedances," IEEE Trans. Power Del., 2007, Vol. 22, No. 2, pp. 1188-1193

[22] Triantafyllidis D. G., Papagiannis G. K. and Labridis D. P., "Calculation of Overhead Transmission Line Impedances: a Finite Element Approach," IEEE Trans. Power Del., 1999, Vol. 14, No. 1, pp. 287-293

[23] Tsiamitros D. A., Papagiannis G. K. and Dokopoulos P. S., "Earth Return Impedances of Conductor Arrangements in Multilayer Soils - Part I: Theoretical Model," IEEE Trans. Power Del., 2008, Vol. 23, No. 4, pp. 2392-2400

[24] Tsiamitros D. A., Papagiannis G. K. and Dokopoulos P. S., "Earth Return Impedances of Conductor Arrangements in Multilayer Soils - Part II: Numerical Results," IEEE Trans. Power Del., 2008, Vol. 23, No. 4, pp. 2401-2408

[25] Wang Y. J and Liu S. J., "A Review of Methods for Calculation of Frequency-Dependent Impedance of Overhead Power Transmission Lines," Proc. Nat. Sci. Counc. ROC(A), 2001, Vol. 25, No. 6, pp. 329-338

[26] Whise W. H., "Effect of Ground Permeability on Ground Return Circuits," Bell. Syst. Tech. J., 1931, Vol. 10, pp. 472-484 
[27] Write Sh. H. and Hall C. F. (Central Station Engineers of the Westinghouse Electric Corporation), "Characteristics of overhead conductors" in Electrical Transmission and Distribution - Reference Book: Pennsylvania East Pittsburgh, 1950, pp. 33-64 